\title{
Capillar Gas Chromatography with Laser Optothermal Detection
}

\author{
V. P. ZHAROV $\dagger$ and S. G. MONTANARI \\ Institute of Spectroscopy, Academy of Science of the USSR, 142092, Moscow \\ Region, Troitzk, USSR
}

(Received 2 February, 1984; in final form 4 May, 1984)

\begin{abstract}
The design of a through-flow laser opto-thermal (OT) detector intended for operation as a selective chromatographic detector in combination with capillary columns is described. The main advantage of such a detector is its relative simplicity and high spatial resolution. A new differential scheme of OT detector which can reduce the background signals of scattered radiation by about $10^{2}$ times is suggested. The threshold sensitivity of OT detector is experimentally estimated to be equal to about $7 \mathrm{ng}$ (ethanol). The higher selectivity of the developed system "gas capillary chromatograph + laser OT spectrometer" has been demonstrated by analyzing a mixture of five atomatic hydrocarbons with the use of a tunable $\mathrm{CO}_{2}$ laser. Recommendations for further modification of the equipment are given.
\end{abstract}

The laser optothermal (OT) method, one of the calorimetric methods, ${ }^{1}$ is based on the detection of thermal oscillations arising in a gas in the process of absorption of modulated resonance laser radiation. As far as its basic characteristics and fields of application are concerned, the OT method is rather close to the opto-acoustic (OA) method based on detecting the acoustic vibrations accompanying thermal ones. This method has gained rather wide acceptance in different fields of science and engineering. ${ }^{2}$ The distinctive feature of the OT method is that it allows studying gas media at low pressures down to $1 \mathrm{mTorr}{ }^{3,4}$ This can be attributed to the detection of absorbed energy when the molecules excited by laser radiation relax directly on the surface of the thermodetector the function of which is usually performed by pyroelectric detectors. A very promising field of application of the OT

$\dagger$ Moscow High Technical School, 107005, Moscow, USSR. 
method is also detecting impurities in gas flows when the laser radiation is passed transversely to the direction of the flow, and the zones of gas heated at radiation absorption are carried by the gas flow to the thermodetector. ${ }^{5}$ The merit of such a scheme of OT detection is increased spatial resolution, i.e., the absorbed energy is detected only in a very small gas space, up to $10^{-2} \mathrm{~cm}^{3}$. This is essential, for example, for express control of impurities in a number of technological processes as well as in chromatography. In Ref. 6, for example, it has been shown that it is promising to use a throughflow OT detector with its threshold absorption sensitivity of $10^{-7} \mathrm{~cm}^{-1}$ as a chromatographic detector in such as combined system as a laser IR spectrometer and a gas chromatograph with the use of packed columns and a $\mathrm{He}-\mathrm{Ne}$ laser with $\lambda=3.39 \mu \mathrm{m}$.

The purpose of the present paper is to continue studying the possibility of applying a through-flow OT detector with capillary chromatographic columns. Such columns, unlike packed ones, make it possible to separate most efficiently composite multicomponent mixtures, but due to very narrow chromatographic peaks (the corresponding volume of carrier gas is 0.1 to $0.01 \mathrm{~cm}^{3}$ ) they require that detectors with small operating volumes should be used. For example, an OA detector, with the volume of its chamber being $2.7 \mathrm{~cm}^{3}$, has proved efficient in operations with packed columns ${ }^{7-9}$ and unfit in operations with capillary columns because of considerable (tens of times) broadening of chromatographic peaks.

Figure 1 presents schematically a measuring setup similar to that described in Ref. 5. The measurements were taken using a "Biochrom1 " $\dagger$ gas chromatograph supplied with a glass capillary column with its parameters: length of $50 \mathrm{~m}$, inside diameter of $0.25 \mathrm{~mm}$, stationary phase is OV-101, carrier gas is He. At the inlet of the column there was a flow dividing value with the division ratio $1: 50$.

At the outlet of the column the carrier gas flow with the separated components of composite mixture were directed into the OT detector using a capillar tube made of stainless steel, its inside diameter $0.5 \mathrm{~mm}$ and length $20 \mathrm{~cm}$. Besides, a flame-ionization detector (FID) (the dashed line in the figure), conventional in chromatography, could be connected to the outlet of the column.

$\dagger$ The device has been developed by the Special design office at the Zelinsky Organic Chemistry Institute, Moscow. 


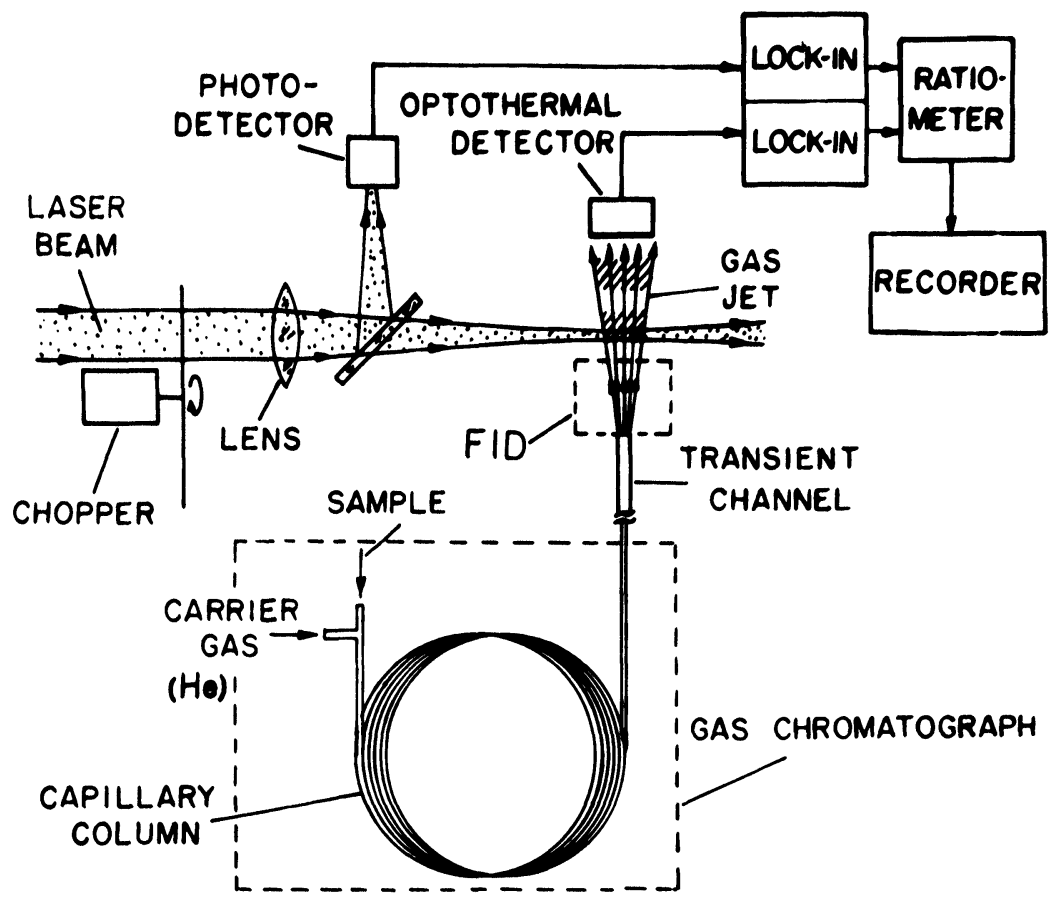

FIGURE 1 Scheme of measuring set up gas chromatograph with laser OT detector.

A CW discretely tunable $\mathrm{CO}_{2}$ laser with its spectral range from 9.2 to $10.8 \mu \mathrm{m}$ and power at each line about $1 \mathrm{~W}$ was used as a radiation source. Its radiation was modulated by a chopper and then directed into the OT detector chamber transversely to the gas flow direction with the use of a lens made of $\mathrm{BaF}_{2}$ with $f=25 \mathrm{~cm}$. Some portion of the radiation was directed to a pyroelectric photodetector by a beam splitter made of $\mathrm{ZnSe}$. The signals from the detectors were amplified by low-noise preamplifiers and after synchronous integration they arrived at the ratiometer and then were detected by a recorder.

In more detail the through-flow OT detector design is shown in Figure 2. The chamber body and the pyroelectric detector holder were made of stainless steel. In the lower part of the body the output end of the connecting capillar tube was fixed. It acted as a nozzle forming a gas jet directed to the surface of the detector. The distance between 


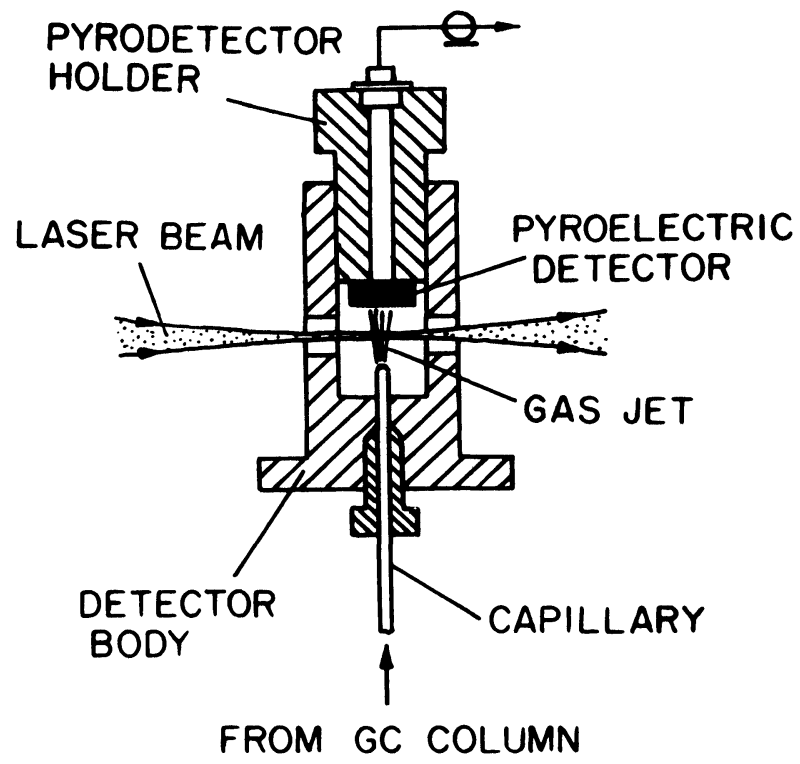

FIGURE 2 Design of through-flow OT detector.

the detector and the nozzle was about $3 \mathrm{~mm}$ and could be varied within small limits. The focused laser radiation was passed through the gas jet at a distance of about $2 \mathrm{~mm}$ from the surface of the thermal detector. The function of such a detector was performed by a pyroelectric detector made in the form of a thin film of pyroactive material $1 \mu \mathrm{m}$ thick placed between two aluminium electrodes sprayed on a glass substratum.

The principle of operation of the described OT detector consists in the following. The carrier gas flow together with the separated components of composite mixture after the chromatographic column is directed onto the surface of the pyroelectric detector with the use of the capillar tube. The laser wavelength is tuned to the intense absorption bands of the components being detected. When these components pass through the space of their interaction with the laser radiation, the fraction of radiation energy which is converted to the thermal energy of medium as a result of nonradiative transitions becomes absorbed. As a consequence, along the gas flow motion a thermal gradient corresponding to the distribution of the detected component in the flow is formed. To make the process of detection more con- 
venient, this thermal distribution is modulated by modulating the laser radiation intensity. The carrier gas flow carries the gas zones with increased temperatures to the pyroelectric detector surface and heats it. As a result, at the detector outlet an electric signal varying with time is formed that is equivalent to the distribution of detected component concentration at column outlet. The advantage of such a combined system, like in Refs. 6-9, is the possibility of identifying components after the column both from the retention time and from the characteristic absorption spectra produced as the laser frequency is changed. Due to the use of capillary columns in this case the potentialities of gas chromatography are maximum as far as its resolution is concerned.

As it can be seen from Figure 2, the described design of OT detector has no windows which would provide isolation of the gas chamber from the environment since there is no principal need in it here. However these windows can be easily introduced if a pressure-tight chamber is needed which may take place in operating with reduced gas pressures at the column outlet or with molecular beams.

As the preliminary experiments showed, it was a serious problem to eliminate the background signals caused by the scattered laser radiation falling directly on the pyroelectric detector surface when a one-element detector was used. Even when the scheme was carefully adjusted, the level of such background signals exceeded the electric noise of the pyroelectric detector by two or three orders. To reduce the effect of such background signals, a differential detector design was developed. Such a detector was fabricated of a conventional detector with its sensitive area $4 \times 4 \mathrm{~mm}^{2}$ in size cut along the diagonal in two rectangular areas which represented two independent pyrodetectors. The detectors were series-connected so that at the same and simultaneous thermal action on the both detectors there were similar signals different in polarity formed on their plates. The signals compensated for each other. But for effective operation of such a differential detectors it was necessary that the useful signal in compensating the background signals of scattered radiation should be detected rather reliably. For this purpose the gas flow was directed only onto one reception area. But because of close distance between the pyrodetectors, that was caused by the wish to provide an approximate equality of the background signals of scattered radiation, some part of the flow arrived at the second detector, too. This, in its turn, 
resulted in essential attenuation of the useful signal by virtue of compensations. Therefore, to get rid of this undesirable effect, a thin plate of $\mathrm{NaCl}(0.5 \mathrm{~mm}$ thick $)$ was glued on the surface of the second detector. This plate was translucent for $\mathrm{CO}_{2}$ laser radiation but prevented thermal contact between the pyrodetector and the gas flow. As a result, the scattered radiation entered the both detectors almost without being attenuated, and the corresponding signals were subtracted, whereas the gas flow with heated zones acted only on one detector and the useful signal almost was not attenuated. The use of such a differential OT detector made it possible to suppress the background signals to the level of electric noise of pyrodetector, i.e., by about $10^{2}$ times. It should be said that at differential connection the total sensitivity of OT detector was somewhat reduced (as compared to the oneelement pyrodetector-that is apparently explained by a decrease of equivalent capacity of pyrodetectors).

The preliminary experiments have shown that, with the carrier gas rate in the capillary column being equal to $2 \mathrm{~cm}^{3} / \mathrm{min}$, the OT detector sensitivity was worse in detecting compounds with comparatively low volatility (with the boiling temperature over $100^{\circ} \mathrm{C}$ ). The chromatographic peaks in this case were broadened as compared to the check measurements carried out with a FID. This variance is apparently caused by the effect of "dead" spaces at the points where the column is linked up with the additional capillar tube as well as by the effect of gas adsorption on the walls of this tube that is not heated enough. As the experiments show, these effects can be easily eliminated by increasing the gas rate through an additional linking capillar. For this purpose an additional flow with its rate up to $30 \mathrm{~cm}^{3} / \mathrm{min}$ was introduced into the carrier gas flow after the column. It should be noted, however, that these steps are not so essential since it is possible to exclude the above-said undesirable effects through proper designing of the OT detector, for example locating it directly at the capillar chromatographic column outlet.

The basic parameters of OT detector were investigated using ethanol with the temperature in the column equal to $80^{\circ} \mathrm{C}$. The $\mathrm{CO}_{2}$ laser was tuned to the line with $\lambda=9.488 \mu \mathrm{m}$ where the absorption factor of ethanol was $6.71 \mathrm{~cm}^{-1} \mathrm{~atm}^{-1}$. ${ }^{10}$

Figure 3 shows the dependence of the OT signal on the magnitude of ethanol sample introduced into the column. It can be seen that within the limits of measurement error this dependence is linear within 


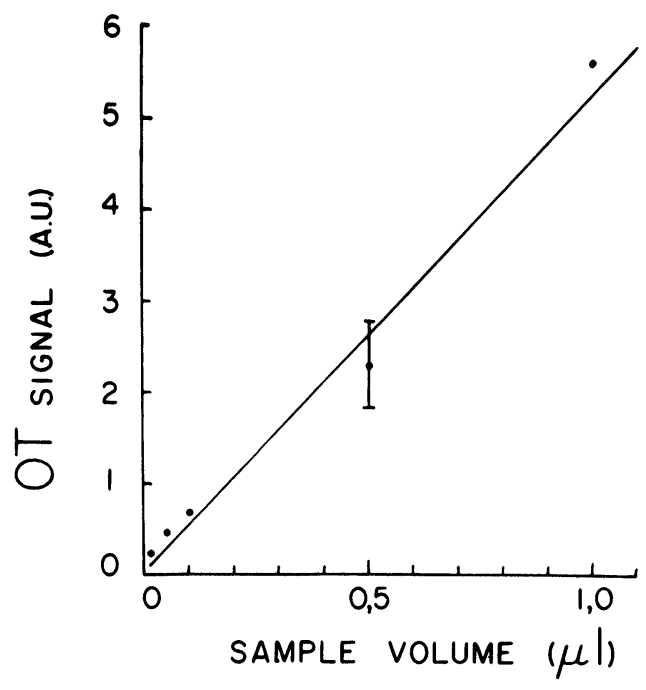

FIGURE 3 Dependence of the OT signal on the quantity of ethanol sample introduced into the capillary gas chromatographic column.

two orders. Some spread of points is mainly due to the error of introduction of the sample using a microinjector.

The extrapolation of the dependence (Figure 3) in the lowconcentration region made it possible to estimate the threshold sensitivity of through-flow OT detector. Under the conditions given above and with the signal-to-noise ratio equal to 1 the threshold sensitivity with respect to absolute amount of substance was about $7 \mathrm{ng}$ and with respect to relative concentration about $6 \mathrm{ng} / \mathrm{cm}^{3}$. To find the threshold sensitivity of OT detector it was necessary to calculate the relative concentration of ethanol corresponding to the chromatographic peak maximum detected as $5 \cdot 10^{-2} \mu \mathrm{l}$ of ethanol was introduced (with allowance made for the flow division at the inlet and the dilution of the carrier gas flow at the outlet of the column). The value of relative concentration $4 \cdot 10^{-2} \%$ obtained with the signal-to-noise ratio equal to $10^{2}$ and the absorption factor known allowed evaluating the OT detector sensitivity on a level of about $5 \cdot 10^{-6} \mathrm{~cm}^{-1}$ with the radiation power at the given line equal to $1 \mathrm{~W}$. The comparatively low sensitivity obtained as compared to the earlier results $\left(\sim 10^{-7} \mathrm{~cm}^{-15-6}\right)$ is explained mainly by a low sensitivity of the differential pyrodetector 
in use. But this sensitivity turned out quite enough for the basic series of studies.

The effect of the detecting space in the OT detector on the chromatographic resolution can be evaluated by comparing the shape of ethanol chromatographic peaks measured under the same conditions using a FID and OT detector (with-out additional gas flow at the column outlet) (Figure 4). It can be seen that the peak half-width at the OT detector outlet is only 1.5 times larger than in the case of the peak measured with a FID. This is quite permissible for a lot of applications. But, as noted above, this broadening is mainly conditioned by the effect of "dead" spaces at the points of connection of capillar tubes which can be eliminated in future designs. Nevertheless, the results demonstrate rather high spatial resolution of OT detector since the gas volume corresponding to the chromatographic peak presented in Figure 4 is equal just to $0.16 \mathrm{~cm}^{3}$.

The practical potentialities of the developed combined system "gas capillary chromatograph + laser OT spectrometer" are demonstrated in Figure 5 by the results of selective analysis of a mixture of five aromatic hydrocarbons carried out using this system. At the top of the figure there is a conventional chromatogram obtained with the use of a FID, at the bottom there is a spectrochromatogram obtained with the use of a five-line OT detector. These results widely demonstrate

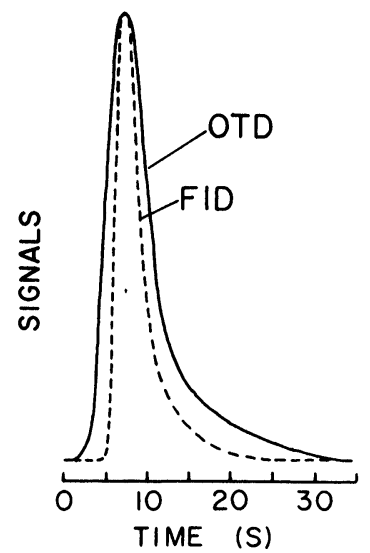

FIGURE 4 Shape of ethanol chromatographic peak measured with an OT detector (solid line) and PID (dashed line). 


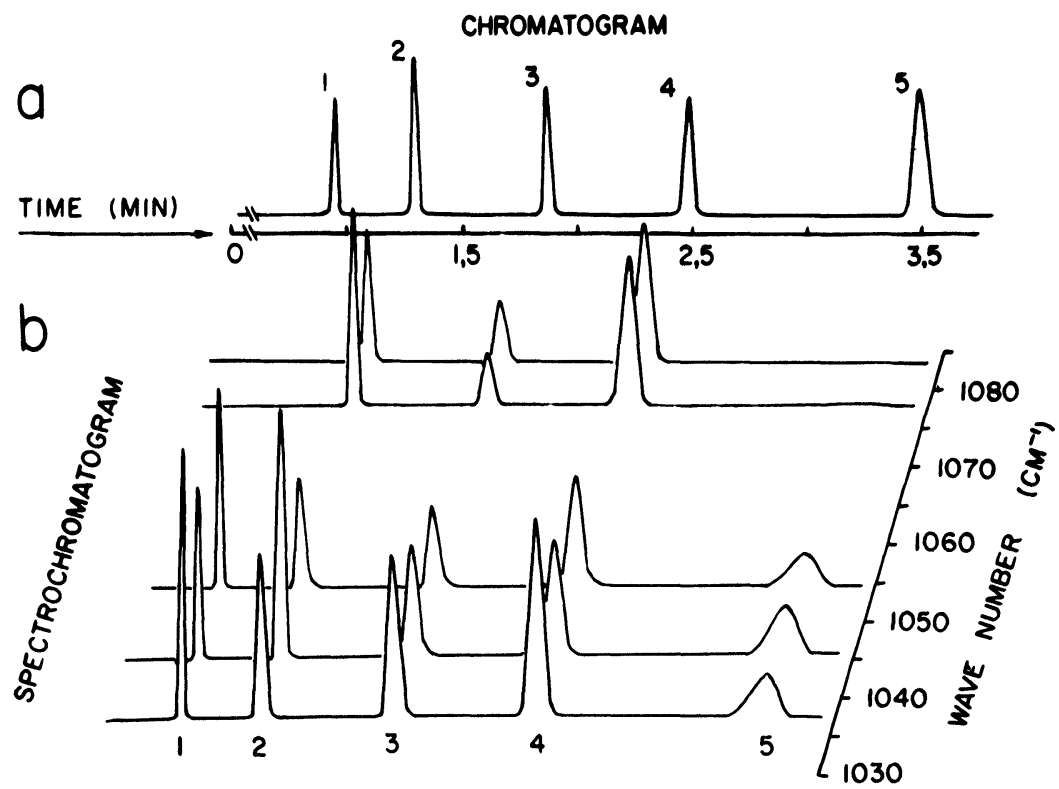

FIGURE 5 (a) the chromatogram of a mixture of 5 aromatic hydrocarbons produced with the use of FID; (b) the spectrochromatogram of the same mixture produced with the use of through-flow five-line OT detector. Composition: (1) benzene; (2) toluene; (3) ethylbenzene; (4) isopropyl benzene; (5) 1,2,3,4-trimethyl benzene.

high (as compared to FID) selectivity of laser OT detector conditioned by differences in the absorption spectra of the given aromatic hydrocarbons.

So, this work is the first to show that it is promising to use a through-flow laser OT detector as a selective chromatographic detector in combination with capillary columns. The basic advantage of such a detector in chromatography in comparison with the OA detector used in Refs. 7-9 is its relative simplicity and increased spatial resolution. The further modification of the described system lies in a further increase of the threshold sensitivity of OT detector by using more sensitive pyroelectrics or bolometers. We hope that it is quite real to attain in practice a threshold absorption sensitivity on the level $10^{-7}$ $10^{-8} \mathrm{~cm}^{-1}$ which is consistent with the concentration threshold of about $0.1 \mathrm{ng}$ of detected substance. This will enable us to apply the OT detector with higher efficiency in a number of tasks already solved 
with the use of a laser OA detector. For example, under different conditions of measurement (multiplex, with "fast" scanning and modulation frequency) it is possible to measure various isomers and impurities, ${ }^{8}$ to identify hydrocarbons in complex mixtures, ${ }^{9}$ to analyze alcohol mixtures, ${ }^{10}$ to identify cyclopentadiene isomers ${ }^{11}$ and fatty acid ethers, ${ }^{12}$ to detect selectively herbicides, ${ }^{13}$, etc.

\section{References}

1. V. P. Zharov, Opto-acoustic method in laser spectroscopy, in: New Spectroscopy Methods (Russian) (Novosibirsk, Nauka, 1982) pp. 126-203.

2. A. Rosencwaig, Photoacoustics and Photoacoustic Spectroscopy (Wiley, New York, 1980).

3. C. Hartung and R. Jurgeit, Kvantovaya Electronika (Russian) 5, 1825 (1978).

4. L. M. Dorozhkin, V. P. Zharov, G. N. Makarov, et al., Pis'ma Zh. Tech. Fiz. (Russian) 6, 979 (1980).

5. V. P. Zharov and S. G. Montanari, in: Proc. III Intern. Conf. on Photoacoustic and Photothermal Spectroscopy (Paris, ESPI, 1983) paper 7.13.

6. V. P. Zharov, V. S. Letokhov and S. G. Montanari, J. Phys. 44, Suppl. N10, C6, 573 (1983).

7. L. B. Kreuzer, Anal. Chem. 50, 597A (1978).

8. V. P. Zharov, S. G. Montanari and V. S. Letokhov, Laser Chem. 1, 163 (1983).

9. V. P. Zharov, V. S. Letokhov, S. G. Montanari and L. M. Tumanova, Dokl. Acad. Nauk SSSR (Russian) 269, 1079 (1983).

10. V. P. Zharov and S. G. Montanari, J. Photoacoustics 1, 355 (1982-1983).

11. V. P. Zharov, S. G. Montanari and L. M. Tumanova, Zhurn. Analit. Himii (Russian) 19, (1984) in press.

12. V. P. Zharov, V. S. Letokhov, S. G. Montanari and L. M. Tumanova, J. Mol. Struct. in press.

13. V. S. Doropheev, V. P. Zharov, S. G. Montanari, et al., Zhurn. Agrohimii (Russian) in press. 